\title{
Correction to: On the Uniform Convergence of Ergodic Averages for $C^{*}$-Dynamical Systems
}

\author{
Francesco Fidaleo®
}

Correction to: Mediterr. J. Math. (2020) 17:125 https://doi.org/10.1007/s00009-020-01553-z

The original article has been published inadvertently with errors in page numbers 6 and 11 .

In page number 6 , the sentence "of the unitary dilation $V_{\varphi, \Phi}$ relative to $\xi \in \mathcal{H}_{\varphi}$. In particular, $\lambda=e^{-\imath \theta} \in \mathbb{T}$ then $\mu_{\xi}(\{\theta\})=\left\|E_{\lambda}^{\varphi, \Phi} \xi\right\|^{2} "$ needs to be changed. The correct sentence is provided below:

of the unitary dilation of $V_{\varphi, \Phi}$ relative to $\xi \in \mathcal{H}_{\varphi}$. In particular, if $\lambda=e^{-\imath \theta} \in$ $\mathbb{T}$ then $\mu_{\xi}(\{\theta\})=\left\|E_{\lambda}^{\varphi, \Phi} \xi\right\|^{2}$.

In Page 11, the sentence "Proposition 4.2. Let $\lambda \in \sigma_{\mathrm{pp}}^{\mathrm{ph}}(\Phi)\{1\}$, and $u \in \mathfrak{A}_{\lambda}$ be an isometry or a coisometry" needs to be changed. The correct sentence is provided below:

Proposition 4.2. Let $\lambda \in \sigma_{\mathrm{pp}}^{\mathrm{ph}}(\Phi) \backslash\{1\}$, and $u \in \mathfrak{A}_{\lambda}$ be an isometry or a co-isometry.

Publisher's Note Springer Nature remains neutral with regard to jurisdictional claims in published maps and institutional affiliations.

Francesco Fidaleo

Dipartimento di Matematica

II Università di Roma "Tor Vergata"

Via della Ricerca Scientifica

00133 Rome

Italy

e-mail: fidaleo@mat.uniroma2.it

The original article can be found online at https://doi.org/10.1007/s00009-020-01553-z. 